\title{
Review of: "Chest MRI of patients with COVID-19"
}

gabriele masselli ${ }^{1}$

1 Sapienza University of Rome

Potential competing interests: The author(s) declared that no potential competing interests exist.

Editorial on Chest MRI of patients with COVID-19

Gabriele Masselli MD PhD

Department of Radiological, Oncological and Pathological Sciences, Sapienza University of Rome, Rome, Italy. Electronic address: gabriele.masselli@uniroma1.it

Chest MR imaging, and particularly the lung, has been historically challenging. The structure of the lung parenchyma is unique and considerably different from other organs and tissues. Major problems result from susceptibility artifacts caused by extensive air-tissue parenchymal interfaces and the low-proton density of the aerated lung, both of which are factors that lead to low signal intensity of the normal lung. Recently, steady state free precession and three-dimensional GRE emerged for visualization of lung parenchymal structures and can increase the signal to-noise ratio within the lung parenchyma.

When compared with CT, three dimensional GRE showed almost perfect agreement for imaging lung nodules or masses, ground-glass opacity, patchy opacity and consolidation, honeycombing and traction bronchiectasis, and substantial agreement in visualizing reticular opacity, emphysema, and bullae (1), Several studies suggest MRI may be comparable to CT in the detection of lung nodules, ground-glass opacity, consolidation, honeycombing, traction bronchiectasis, and reticular changes $(1,2)$.

T2-weighted turbo spin-echo turbo inversion recovery magnitude (T2W TSE-TIRM) was found to resolve lesions more brightly than the other sequences studied, likely highlighting areas of edema secondary to parenchymal inflammatory changes..

Yet, the intrinsic properties of the lung parenchyma are in fact advantages when imaging pathology of the alveolar spaces, as GGOs and consolidation appear hyperintense relative to the surrounding tissues secondary to exudative fluid accumulation and increased proton density.

Moreover MRI with DWI identified lung inflammatory lesions with an accuracy similar to that obtained using HRCT (3).

In this issue of Magnetic Resonance Imaging Yu A. Vasilev et al evaluated the possibility of using chest 
magnetic resonance imaging (MRI) as an alternative, non-ionizing method for detecting pneumonia in patients with COVID (4).

They demonstrated visualization of GGOs, consolidation, reticulation, and a reverse halo sign on multiple MRI sequences with a good accuracy in MRI detection of GGOs or consolidative lesions versus conventional CT.

Their study reports substantial or excellent intermethod agreement for detecting typical findings of COVID19 pneumonia, including pure GGOs, pure consolidation, and GGOs with consolidation; however, only fair to moderate lesion-based intermethod agreement was observed for the assessment of secondary signs, including pseudocavities, crazy paving pattern, and air bronchograms. Overall, these results are consistent with existing literature in related pulmonary infections suggesting MRI adequately distinguishes between different stages of parenchymal infiltration in head-to-head comparisons with chest CT.

Current guidelines from the American College of Radiology recommend limiting the use of MRI in confirmed or suspected SARS-CoV-2-positive patients.

The results of this study showed that in case of CT is not available, it is advisable to conduct a chest MRI for patients with suspected or confirmed COVID19.

For patient groups in whom excessive or repeated exposure to ionizing radiation should be avoided, pulmonary MRI may yet provide a viable alternative.

Though not considered routine for evaluating suspected lower respiratory tract infections, pulmonary MRI may provide a viable alternative for imaging high-risk patient groups such as pregnant women and children in whom exposure to ionizing radiation should be avoided.

Moreover pulmonary contrast enhanced magnetic resonance angiography (CE-MRA) has an accuracy similar to CT for the detection of pulmonary embolism (PE), and it can be useful for assessing COVID patients with suspected PE (5).

MRI has been demonstrated to be a reliable tool in the follow-up of COVID-19 patients-being, notably, a radiation-free, minimally invasive imaging modality.

DWI MRI might constitute a promising imaging biomarker in the evaluation of a pulmonary active inflammatory process, which might influence decision-making in the patient's clinical management course, and the long-term impact on lung health status.

In conclusion this study, according with other recent studies, supports the use state-of-the-art lung MRI as an alternative to $\mathrm{CT}$, in particular for pediatric patients and pregnant women, and it can be helpful for repeated imaging follow -up in patients with COVID infection

1. Ohno Y, Koyama H, Yoshikawa T, et al. Pulmonary high-resolution ultrashort TE MR imaging: Comparison with thin-section standard- and low-dose computed tomography for the assessment of pulmonary parenchyma diseases. J Magn Reson Imaging 2016;43(2):512-532.

2. P Torkian, Hamid Rajebi , Taraneh Zamani, Naghi Ramezani, Pejman Kiani Shahram Akhlaghpoor 
Magnetic resonance imaging features of coronavirus disease 2019 (COVID-19) pneumonia: The first preliminary case series. Clin Imaging . 2021 Jan;69:261-265. doi: 10.1016/j.clinimag.2020.09.002.

3. O Ates, Taydas $\mathrm{O}$, Dheir $\mathrm{H}$ et al. Thorax Magnetic Resonance Imaging Findings in Patients with Coronavirus Disease (COVID-19) Acad Radiol . 2020 Oct;27(10):1373-1378.

4. Yu A. Vasilev a,1, K.A. Sergunova a,1, A.V. Bazhin et al Chest MRI of patients with COVID-19. Magnetic Resonance Imaging 79 (2021) 13-19

5. Masselli G, Almberger M, Tortora A, Capoccia L, Dolciami M, D'Aprile MR, Valentini C, Avventurieri G, Bracci S, Ricci P. Role of CT angiography in detecting acute pulmonary embolism associated with COVID-19 pneumonia. Radiol Med. 2021 Dec;126(12):1553-1560. 